\title{
Serum osteocalcin levels in overweight children
}

\author{
Seok Bin Oh, MD, \\ Won Young Lee, MD, \\ Hyo-Kyoung Nam, MD, PhD, \\ Young-Jun Rhie, MD, PhD, \\ Kee-Hyoung Lee, MD, PhD
}

Department of Pediatrics, Korea University College of Medicine, Seoul, Korea
Purpose: Bone plays a role in glucose metabolism through the release of uncarboxylated osteocalcin into the systemic circulation. The identified novel roles for osteocalcin include increasing insulin secretion and sensitivity, energy expenditure, reduction of fat mass, and mitochondrial proliferation and functional enhancement. This study aimed to determine serum osteocalcin levels in overweight children and to investigate the relationships of osteocalcin with glucose metabolism and insulin sensitivity.

Methods: After overnight fasting, serum osteocalcin levels were measured in overweight $(n=50)$ children between 6.0 and 12.9 years of age and nonoverweight controls $(n=60)$. Height, weight, fasting serum glucose, insulin, alkaline phosphatase, total cholesterol, and 25 hydroxy vitamin $\mathrm{D}_{3}\left(25(\mathrm{OH}) \mathrm{Vit}_{3}\right)$ were also measured in all subjects.

Results: There were significant differences in serum osteocalcin levels between the overweight and control groups $(64.00 \pm 20.44$ vs. $89.56 \pm 28.63, P<0.001)$. Serum osteocalcin levels were inversely correlated with body mass index (BMI) $(r=-0.283$, $P=0.003)$, weight standard deviation score (SDS) $(r=-0.222, P=0.020)$, BMI SDS ( $r=-$ $0.297, P=0.002)$, insulin $(r=-0.313, P=0.001)$, and homeostasis model assessment of insulin resistance (HOMA-IR) index $(r=-0.268, P=0.005)$. In the subsequent multiple regression analyses, BMI, HOMA-IR, and age were determined to be independent predicting factors for serum osteocalcin.

Conclusion: Our findings showed associations of serum osteocalcin with glucose metabolism and insulin sensitivity in overweight children, but we could not establish a causal relationship.

Keywords: Overweight, Glucose, Metabolism, Osteocalcin

\section{Introduction}

Obesity is one of the most serious health problems globally, especially among children and adolescents. Over the past several years, the prevalence of obesity in children and adolescents has increased rapidly. ${ }^{1,2)}$ The prevalence of obesity in Korean children increased from $6.8 \%$ in 1998 to $10.0 \%$ in 2013 . $^{3)}$ The consequences of obesity in childhood include type 2 diabetes mellitus, obesity in adulthood, and increased incidences of metabolic syndrome and cardiovascular disease as adults. ${ }^{4-7)}$ Thus, the early detection and prevention of obesity in children is essential.

Bone has been recognized as a target of hormones that affect calcium and phosphorus homeostasis and bone structure. However, the role of bone as an endocrine organ has recently been revealed, and recent studies have shown that bone plays a metabolic role when uncarboxylated osteocalcin is released into the systemic circulation. ${ }^{8)}$ Osteocalcin is a hormone found in bone that is secreted by osteoblasts. Osteocalcin plays a role in the body's metabolic regulation and bone building. ${ }^{9)}$ Recent studies revealed metabolic roles for osteocalcin, which include promoting insulin secretion and sensitivity, energy expenditure, decreasing fat mass, and mitochondrial proliferation and functional enhancement. ${ }^{9)}$ In addition, osteocalcin functions as a biochemical marker for bone formation and metabolic regulation and acts as a 
hormone regulating glucose metabolism and fat mass. ${ }^{8,10,11)}$

Some studies have reported that osteocalcin is independently influenced by body mass index (BMI). ${ }^{12,13)}$ A metabolic role of osteocalcin in obesity and insulin resistance has also been suggested, and low osteocalcin levels have been measured in overweight people. ${ }^{14-16)}$

Recently, several studies have been conducted regarding the association between serum osteocalcin levels and glucose metabolism. ${ }^{8-11)}$ In some human and animal studies, osteocalcin has been shown to affect the secretion and sensitivity of insulin and to reduce the deposition of fat. ${ }^{8,14-16)}$ Osteocalcin also increases the secretion of adiponectin from adipose tissue and has been shown to be inversely correlated with obesity. ${ }^{8,14-16)}$

However, in children, few data concerning an association between serum osteocalcin levels and glucose metabolism are available. ${ }^{17,18)}$ Therefore, this study aimed to determine the serum osteocalcin levels of overweight children and to investigate osteocalcin's relationship with glucose metabolism and insulin sensitivity.

\section{Materials and methods}

\section{Subjects}

The subjects consisted of 2 groups: overweight children and their nonoverweight controls. Overweight subjects aged between 6.0 and $12.9(n=50)$ were recruited from an outpatient clinic at Korea University Ansan Hospital from September 2016 to June 2017. The subjects had a BMI above the 85th percentile for their age and sex according to 2007 Korean National Growth Charts. Overweight patients who had genetic causes of obesity or endocrine disorders such as diabetes were excluded from this study.

The control group $(n=60)$ consisted of age-matched nonoverweight children (25th percentile $\leq \mathrm{BMI}<75$ th percentile according to 2007 Korean National Growth Charts). These subjects were also recruited as volunteers from the outpatient clinic at Korea University Ansan Hospital.

\section{Data collection}

The following demographic and clinical data were collected at the first visit: age, sex, height, and body weight. Height was measured using a rigid stadiometer, and weight was measured to the nearest $0.1 \mathrm{~kg}$ using a calibrated balance scale. BMI was calculated as weight in kilograms divided by height in meters squared. Standard deviation score (SDS) of height, weight, and BMI by age and sex was obtained according to 2007 Korean National Growth Charts. ${ }^{19)}$ Fasting serum glucose, insulin, alkaline phosphatase (ALP), total cholesterol, 25 hydroxy vitamin $\mathrm{D}_{3}\left(25(\mathrm{OH}) \mathrm{VitD}_{3}\right)$, and osteocalcin levels were measured. Serum osteocalcin levels in both the overweight and control groups were measured using an electrochemiluminescence immunoassay (Roche Diagnostics, Indianapolis, IN, USA). Insulin resistance was estimated by the homeostasis model assessment of insulin resistance (HOMA-IR) index [ Insulin $(\mu \mathrm{IU} / \mathrm{mL}) \times$ glucose $(\mathrm{pmol} / \mathrm{L}) / 22.5]$.

\section{Statistical analysis}

The IBM SPSS Statistics ver. 20.0 (IBM Co., Armonk, NY, USA) was used for statistical analyses. Data are expressed as mean \pm standard deviation. To compare between the overweight and control groups, we used an independent t-test. To assess relationships between serum osteocalcin levels and clinical characteristics such as age, height, weight, BMI, height SDS, weight SDS, BMI SDS, fasting glucose, serum insulin, HOMAIR, ALP, total cholesterol, and $25(\mathrm{OH}) \mathrm{VitD}_{3}$, we used a Pearson correlation test; $P$-values under 0.05 were considered statistically significant. A multiple regression analysis was used to determine the factors associated with osteocalcin concentration.

\section{Results}

The clinical and metabolic characteristics of both subgroups are shown in Table 1. There were no significant differences in sex distribution, age, fasting glucose level, ALP, total cholesterol, and $25(\mathrm{OH}) \mathrm{VitD}_{3}$ level between the 2 groups. In the overweight group, weight $(P<0.001)$, height $(P=0.004)$, BMI $(P<0.001)$, height SDS $(P<0.001)$, weight SDS $(P<0.001)$, BMI SDS $(P<0.001)$, serum insulin level $(P=0.003)$, and HOMAIR $(P=0.008)$ were significantly higher than the control group. However, serum osteocalcin levels were significantly lower in the overweight group $(64.00 \pm 20.44$ vs. $89.56 \pm 28.63, P<0.001)$.

Table 2 shows laboratory data establishing the correlation of different characteristics with osteocalcin concentration. Serum osteocalcin levels were inversely correlated with BMI $(r=-0.283$,

Table 1. Comparison of clinical and laboratory data between overweight and control groups

\begin{tabular}{|c|c|c|c|}
\hline Variable & $\begin{array}{l}\text { Overweight } \\
(n=50)\end{array}$ & $\begin{array}{l}\text { Control } \\
(n=60)\end{array}$ & $P$-value \\
\hline Sex, male:female & $23: 27$ & $29: 31$ & 0.807 \\
\hline Age (yr) & $9.34 \pm 1.38$ & $9.72 \pm 1.76$ & 0.209 \\
\hline Height (cm) & $138.9 \pm 9.8$ & $132.3 \pm 13.6$ & 0.004 \\
\hline Weight (kg) & $47.6 \pm 13.6$ & $29.9 \pm 9.2$ & $<0.001$ \\
\hline BMI $\left(\mathrm{kg} / \mathrm{m}^{2}\right)$ & $24.3 \pm 4.2$ & $16.7 \pm 2.2$ & $<0.001$ \\
\hline Height SDS & $1.07 \pm 1.23$ & $-0.44 \pm 1.04$ & $<0.001$ \\
\hline Weight SDS & $1.90 \pm 0.81$ & $-0.56 \pm 1.04$ & $<0.001$ \\
\hline BMI SDS & $1.93 \pm 0.63$ & $-0.46 \pm 1.07$ & $<0.001$ \\
\hline Fasting glucose (mg/dL) & $90.9 \pm 6.9$ & $90.8 \pm 9.3$ & 0.986 \\
\hline Insulin (mU/L) & $21.47 \pm 11.66$ & $15.48 \pm 8.34$ & 0.003 \\
\hline HOMA-IR & $4.84 \pm 2.78$ & $3.57 \pm 2.17$ & 0.008 \\
\hline $\operatorname{ALP}(I U / L)$ & $284.4 \pm 69.7$ & $258.1 \pm 86.0$ & 0.087 \\
\hline Total cholesterol (mg/dL) & $166.4 \pm 32.2$ & $170.7 \pm 28.7$ & 0.458 \\
\hline $25(\mathrm{OH}) \mathrm{Vit}_{3}(\mathrm{ng} / \mathrm{mL})$ & $23.07 \pm 7.06$ & $25.09 \pm 8.87$ & 0.252 \\
\hline Osteocalcin (ng/mL) & $64.00 \pm 20.44$ & $89.56 \pm 28.63$ & $<0.001$ \\
\hline
\end{tabular}

Values are presented as mean \pm standard deviation.

$\mathrm{BMI}$, body mass index; SDS, standard deviation score; HOMA-IR, homeostasis model assessment of insulin resistance index; ALP, alkaline phosphatase; $25(\mathrm{OH}) \mathrm{VitD}_{3}, 25$ hydroxy vitamin $\mathrm{D}_{3}$. 
$P=0.003)$, weight SDS $(r=-0.222, P=0.020)$, BMI SDS $(r=-0.297$, $P=0.002)$, insulin $(r=-0.313, P=0.001)$, and HOMA-IR $(r=-0.268$, $P=0.005)$. Age was positively correlated with serum osteocalcin level ( $r=0.202, P=0.034)$. However, height, weight, height SDS, fasting glucose, ALP, total cholesterol, and $25(\mathrm{OH}) \mathrm{VitD}_{3}$ levels were shown to have no relationship with serum osteocalcin $(P>0.05)$.

The multivariate analysis showed that BMI (coefficient $\beta=$ $0.258, P=0.006$ ), HOMA-IR (coefficient $\beta=-0.388, P<0.001$ ), and age (coefficient $\beta=0.285, P=0.003$ ) remained as independent predictors of serum osteocalcin. However, fasting glucose, ALP, and total cholesterol had no independent relationships with serum osteocalcin (Table 3).

\section{Discussion}

This study was the first to assess the relationship between serum osteocalcin level and being overweight in the Korean pediatric population. In our study, serum osteocalcin level was significantly lower in overweight children. Furthermore, serum osteocalcin level was inversely correlated with BMI, weight SDS, serum insulin level, and HOMA-IR. These results suggest that

Table 2. Correlation of clinical and laboratory data with osteocalcin concentration

\begin{tabular}{lcr}
\hline Variable & $r$ & $P$-value \\
\hline Age & 0.202 & 0.034 \\
Height & 0.182 & 0.057 \\
Weight & -0.119 & 0.214 \\
Body mass index & -0.283 & 0.003 \\
Height SDS & -0.010 & 0.915 \\
Weight SDS & -0.222 & 0.020 \\
BMI SDS & -0.297 & 0.002 \\
Fasting glucose & 0.094 & 0.327 \\
Insulin & -0.313 & 0.001 \\
HOMA-IR & -0.268 & 0.005 \\
ALP & 0.084 & 0.384 \\
Total cholesterol & -0.098 & 0.313 \\
25(OH)VitD & -0.048 & 0.641 \\
\hline Person corration coffcients & sh for associtis
\end{tabular}

Pearson correlation coefficients are shown for associations with serum osteocalcin concentration.

SDS, standard deviation score; BMI, body mass index; HOMA-IR, homeostasis model assessment of insulin resistance index; ALP, alkaline phosphatase; $25(\mathrm{OH}) \mathrm{Vit}_{3}, 25$ hydroxy vitamin $\mathrm{D}_{3}$.

Table 3. Results of multiple linear regression analysis of factors associated with osteocalcin concentration

\begin{tabular}{lcc}
\hline Variable & $\beta$ & $P$-value \\
\hline Age & 0.285 & 0.003 \\
BMI & -0.258 & 0.006 \\
Fasting glucose & 0.171 & 0.087 \\
HOMA-IR & -0.388 & $<0.001$ \\
ALP & 0.076 & 0.397 \\
Total cholesterol & -0.124 & 0.162 \\
\hline
\end{tabular}

$\overline{B M I}$, body mass index; HOMA-IR, homeostasis model assessment of insulin resistance index; ALP, alkaline phosphatase. being overweight is inversely associated with serum osteocalcin level, but we could not establish a causal relationship.

Osteocalcin's roles in bone formation have been known for decades. ${ }^{13,20)}$ However, in recent studies, its metabolic regulatory roles have been revealed. These roles include regulation of serum glucose and fat deposition as well as essential roles in glucose metabolism. ${ }^{8)}$ A recent report by Pittas et al. ${ }^{21)}$ showed that serum osteocalcin level was inversely correlated with fasting serum glucose, fasting insulin, insulin resistance, and fat mass. In adults, an inverse correlation between serum osteocalcin level and BMI has been demonstrated in many studies. ${ }^{22-24)}$ In addition, Reinehr and $\operatorname{Roth}^{17)}$ analyzed osteocalcin, adiponectin, leptin, and HOMA-IR in obese Caucasian children and showed that these children had significantly lower serum osteocalcin levels than the normal controls. In concordance with these studies, our study showed inverse correlations of serum osteocalcin level with BMI, insulin, and HOMA-IR in children.

However, the exact mechanisms of osteocalcin in glucose metabolism are unclear. In experiments with mice, Lee et al. ${ }^{8)}$ discovered that serum osteocalcin inhibits insulin secretion, decreases beta-cell proliferation, and increases insulin resistance. Since then, several different studies have examined the relationship between serum osteocalcin and glucose metabolism. The adipocytokine leptin has important effects on bone metabolism, and osteocalcin is thought to regulate insulin sensitivity through adiponectin. ${ }^{8,25,26)}$

In our study, serum osteocalcin levels were inversely correlated with HOMA-IR in the Pearson correlation test and multiple regression analysis. Previous studies conducted in children and adults showed an inverse correlation between serum osteocalcin level and insulin resistance. ${ }^{89}$ Im et al. reported that osteocalcin was an independent factor associated with serum glucose and glycosylated hemoglobin in postmenopausal women. ${ }^{14,23)}$ These findings have suggested that osteocalcin plays a crucial role not only in bone remodeling but also in glucose metabolism.

In recent studies, leptin and adiponectin have been considered as having a vital role in the glucose metabolism of serum osteocalcin. ${ }^{14-16)}$ Reinehr and Roth ${ }^{17)}$ reported that osteocalcin level is negatively associated with leptin in German children, but not associated with adiponectin. However, associations between osteocalcin and leptin or adiponectin were not included in our study.

In addition, our study was conducted in overweight children without diabetes. In this study, insulin resistance was increased and serum osteocalcin level was decreased even in overweight children without diabetes. These results demonstrate that there were changes in glucose metabolism in overweight children, even those without diabetes.

Our study had several limitations. For example, the small number of subjects in both the test and control groups was a limitation. In addition, we could not completely control for all factors affecting the results because the mechanism of osteocalcin in glucose metabolism is not fully understood. Also, this study had a cross-sectional design and, therefore, could not provide a causal relationship between serum osteocalcin and being overweight. 
In conclusion, serum osteocalcin level was associated with glucose metabolism and insulin sensitivity in our study of overweight children, but we could not establish a causal relationship.

Larger longitudinal studies are required to validate our results in addition to studies aimed at revealing the mechanism of serum osteocalcin in glucose metabolism.

\section{Ethical statement}

This study was approved by the Institutional Review Board of Korea University Ansan Hospital, Korea (IRB No. 2018AS0165), and written informed consent was obtained from all subjects and their parents.

\section{Conflict of interest}

No potential conflict of interest relevant to this article was reported.

\section{References}

1. Park YS, Lee DH, Choi JM, Kang YJ, Kim CH. Trend of obesity in school age children in Seoul over the past 23 years. Korean J Pediatr 2004;47:247-57.

2. Skinner AC, Ravanbakht SN, Skelton JA, Perrin EM, Armstrong SC. Prevalence of obesity and severe obesity in US children, 1999-2016. Pediatrics 2018;141(3). pii: e20173459. https://doi.org/0.1542/peds.2017-3459.

3. Ha KH, Kim DJ. Epidemiology of childhood obesity in Korea. Endocrinol Metab 2016;31:510-8.

4. Skinner AC, Perrin EM, Moss LA, Skelton JA. Cardiometabolic risks and severity of obesity in children and young adults. N Engl J Med 2015;373:1307-17.

5. Chen F, Wang Y, Shan X, Cheng H, Hou D, Zhao X, et al. Association between childhood obesity and metabolic syndrome: evidence from a large sample of Chinese children and adolescents. PLoS One 2012;7:e47380.

6. Chung JY, Kang HT, Shin YH, Lee HR, Park BJ, Lee YJ. Prevalence of metabolic syndrome in children and adolescents - the recent trends in South Korea. J Pediatr Endocrinol Metab 2013;26:105-10.

7. Daniels SR. Complications of obesity in children and adolescents. Int J Obes (Lond) 2009;33 Suppl 1:S60-5.

8. Lee NK, Sowa H, Hinoi E, Ferron M, Ahn JD, Confavreux $\mathrm{C}$, et al. Endocrine regulation of energy metabolism by the skeleton. Cell 2007;130:456-69.

9. Kim YS, Paik IY, Rhie YJ, Suh SH. Integrative physiology: defined novel metabolic roles of osteocalcin. J Korean Med Sci 2010;25:985-91.

10. Hauschka PV, Lian JB, Cole DE, Gundberg CM. Osteocalcin and matrix Gla protein: vitamin K-dependent proteins in bone. Physiol Rev 1989;69:990-1047.

11. Hauschka PV, Wians FH Jr. Osteocalcin-hydroxyapatite interaction in the extracellular organic matrix of bone. Anat Rec 1989;224:180-8.
12. Delmas PD. Biochemical markers of bone turnover for the clinical investigation of osteoporosis. Osteoporos Int 1993;3 Suppl 1:81-6.

13. Szulc P, Seeman E, Delmas PD. Biochemical measurements of bone turnover in children and adolescents. Osteoporos Int 2000;11:281-94.

14. Im JA, Yu BP, Jeon JY, Kim SH. Relationship between osteocalcin and glucose metabolism in postmenopausal women. Clin Chim Acta 2008;396:66-9.

15. Kindblom JM, Ohlsson C, Ljunggren O, Karlsson MK, Tivesten A, Smith U, et al. Plasma osteocalcin is inversely related to fat mass and plasma glucose in elderly Swedish men. J Bone Miner Res 2009;24:785-91.

16. Zhou M, Ma X, Li H, Pan X, Tang J, Gao Y, et al. Serum osteocalcin concentrations in relation to glucose and lipid metabolism in Chinese individuals. Eur J Endocrinol 2009;161:723-9.

17. Reinehr T, Roth CL. A new link between skeleton, obesity and insulin resistance: relationships between osteocalcin, leptin and insulin resistance in obese children before and after weight loss. Int J Obes (Lond) 2010;34:852-8.

18. Kim GS, Jekal Y, Kim HS, Im JA, Park JY, Chu SH. Reduced serum total osteocalcin is associated with central obesity in Korean children. Obes Res Clin Pract 2014;8:e201-98.

19. Moon JS, Lee SY, Nam CM, Choi JM, Choe BK, Seo JW, et al. 2007 Korean National Growth Charts: review of developmental process and an outlook. Korean J Pediatr 2008;51:1-25.

20. Ducy P, Desbois C, Boyce B, Pinero G, Story B, Dunstan $\mathrm{C}$, et al. Increased bone formation in osteocalcin-deficient mice. Nature 1996;382:448-52.

21. Pittas AG, Harris SS, Eliades M, Stark P, Dawson-Hughes B. Association between serum osteocalcin and markers of metabolic phenotype. J Clin Endocrinol Metab 2009;94:827-32.

22. Hinton PS, Rector RS, Thomas TR. Weight-bearing, aerobic exercise increases markers of bone formation during shortterm weight loss in overweight and obese men and women. Metabolism 2006;55:1616-8.

23. Holecki M,Zahorska-Markiewicz B, Janowska J, Nieszporek T, Wojaczyńska-Stanek K, Zak-Gołab A, et al. The influence of weight loss on serum osteoprotegerin concentration in obese perimenopausal women. Obesity (Silver Spring) 2007;15:1925-9.

24. Pereira FA, de Castro JA, dos Santos JE, Foss MC, Paula FJ. Impact of marked weight loss induced by bariatric surgery on bone mineral density and remodeling. Braz J Med Biol Res 2007;40:509-17.

25. Reid IR, Cornish J, Baldock PA. Nutrition-related peptides and bone homeostasis. J Bone Miner Res 2006;21:495-500.

26. Weyer C, Funahashi T, Tanaka S, Hotta K, Matsuzawa Y, Pratley RE, et al. Hypoadiponectinemia in obesity and type 2 diabetes: close association with insulin resistance and hyperinsulinemia. J Clin Endocrinol Metab 2001;86:19305. 